\title{
Exponential effort estimation model using unadjusted function points.
}

\begin{abstract}
In this study, we present a window-based exponential effort estimation model to predict the effort required in terms of man days by using Unadjusted Function Point (UFP) size measure and eliminate the usage of General Systems Characteristics (GSCs). A very comprehensive statistical analysis and test was carried out on large amount of quality project data in the International Software Benchmarking Standard Group (ISBSG) Release 9 dataset, which were collected by the International Function Points User Group (IFPUG) count approach in the process of model development. The effectiveness of the model was examined and reported in this study.
\end{abstract}

Keyword: Functional size measure; Effort estimation model; Unadjusted function point; Regression analysis. 